Artificial piezoelectric grass for energy harvesting from turbulence-induced vibration

This article has been downloaded from IOPscience. Please scroll down to see the full text article.

2012 Smart Mater. Struct. 21105024

(http://iopscience.iop.org/0964-1726/21/10/105024)

View the table of contents for this issue, or go to the journal homepage for more

Download details:

IP Address: 141.211.173.82

The article was downloaded on 25/06/2013 at 20:35

Please note that terms and conditions apply. 


\title{
Artificial piezoelectric grass for energy harvesting from turbulence-induced vibration
}

\author{
J D Hobeck and D J Inman \\ Department of Aerospace Engineering, University of Michigan, Ann Arbor, MI 48109, USA \\ E-mail: jdhobeck@umich.edu
}

Received 27 February 2012, in final form 13 July 2012

Published 20 August 2012

Online at stacks.iop.org/SMS/21/105024

\begin{abstract}
The primary objective of this research is to develop a deploy-and-forget energy harvesting device for use in low-velocity, highly turbulent fluid flow environments i.e. streams or ventilation systems. The work presented here focuses on a novel, lightweight, highly robust, energy harvester design referred to as piezoelectric grass. This biologically inspired design consists of an array of cantilevers, each constructed with piezoelectric material. When exposed to proper turbulent flow conditions, these cantilevers experience vigorous vibrations.

Preliminary results have shown that a small array of piezoelectric grass was able to produce up to $1.0 \mathrm{~mW}$ per cantilever in high-intensity turbulent flow having a mean velocity of $11.5 \mathrm{~m} \mathrm{~s}^{-1}$. According to the literature, this is among the highest output achieved using similar harvesting methods. A distributed parameter model for energy harvesting from turbulence-induced vibration will be introduced and experimentally validated. This model is generalized for the case of a single cantilever in turbulent cross-flow. Two high-sensitivity pressure probes were needed to perform spectral measurements within various turbulent flows. The design and performance of these probes along with calibration and measurement techniques will be discussed.
\end{abstract}

(Some figures may appear in colour only in the online journal)

\section{Introduction}

Turbulence-induced vibration (TIV) is generally considered undesirable, and is a phenomenon that if not properly anticipated can lead to catastrophic structural failure. From an energy harvesting perspective however, these types of vibrations have been found to be quite valuable [1]. Unlike vortex-induced vibration, flutter, or acoustic resonance, where vibrations can be minimized or essentially eliminated by design, TIV is inevitable in dynamic fluid environments [2]. Analytical investigations of TIV have been performed for decades; however, it was not until very recently that there has been interest shown in developing devices that can generate useful power from these vibrations.

A recent experimental study was done in which a PVDF cantilever beam with dimensions $30 \mathrm{~mm} \times 16 \mathrm{~mm} \times 0.2 \mathrm{~mm}$ was placed with its length parallel to turbulent boundary layer flow [3]. Rather than using pure vortex shedding, flutter, or related fluidelastic phenomena, this turbulent boundary layer experiment was the first reported energy harvesting study conducted where turbulence was the primary excitation mechanism. The maximum power output was nearly $0.06 \mu \mathrm{W}$ in a free-stream velocity of approximately $11.0 \mathrm{~m} \mathrm{~s}^{-1}$. This is an extremely low output considering the same harvester was shown to produce more than $4 \mu \mathrm{W}$ when placed in the vortex street of a cylinder in air with a velocity of only $7.23 \mathrm{~m} \mathrm{~s}^{-1}$ [3]. The drastic reduction in power output is to be expected given the parameters chosen; however, the power output potential of TIV energy harvesting should not be regarded as insignificant.

According to the literature, previous investigations on the topic of TIV energy harvesting do not exist. This paper presents the first experimentally validated modeling approach for TIV energy harvesting and will focus on experimental techniques and results. 


\subsection{Existing fluid flow harvesting methods}

Traditional harvester designs include those with turbines or propellers, and their motion typically involves directional rotation of a shaft. Electric energy is produced via electromagnetism [4] or even piezoelectricity [5]. Turbulence causes an unsteady, poorly correlated distribution of forces across the surfaces of these devices which can significantly diminish their performance. Another disadvantage of these traditional harvester designs with regard to long-term survival in natural or uncontrolled environments is their susceptibility to damage. For example: debris may come into contact with the blades or get entangled around a rotating shaft which can damage or jam the device, rendering it useless.

Piezoelectric energy harvesters with biologically inspired designs have been explored. An artificial kelp design was proposed by Pankonien and Ounaies for wave or tidal flow [6]. Hobbs and $\mathrm{Hu}$ presented a tree-inspired design for vortex-induced vibration harvesting [7]. While both [6, 7] have a similar design concept to the harvester presented in this paper, the form of excitation used to estimate power output is appropriate for either bulk fluid motion or vortex-induced vibration rather than for TIV.

Flutter or flapping mechanisms operate on the principal of fluidelastic instability. Bryant and Garcia were among the first to propose the concept of energy harvesting from vibrations caused by aerodynamic flutter $[8,9]$. A feasibility study was performed on the concept of energy harvesting from elastic bluff body wake galloping by Jung et al [10]. Pitch and plunge airfoil flutter dynamics were discussed and numerically simulated by Shimizu et al [11] while an experimentally validated analytical model with application to piezoaeroelastic energy harvesting was presented by De Marqui et al [12]. When introduced to highly turbulent flow, periodic separation and reattachment of flow on the structure becomes sporadic and less coupled to the dynamics of the structure. These conditions may cause intermittent flutter or none at all.

Extensive research has been done in the area of generating power from vortex-induced vibration. A rigid cylinder supported by springs on both ends while subject to low-velocity $\left(0.25 \mathrm{~m} \mathrm{~s}^{-1}\right)$ cross-flow was shown to oscillate perpendicular to the flow at large amplitudes [13]. This motion was converted into electrical energy by connecting the cylinders to generators via a gear-belt system. A smaller, solid-state design was presented by Pobering et al, and consisted of a PZT cantilever mounted to the downstream side of a bluff body [14]. As vortices shed off the bluff body and traveled down the length of the cantilever they produced periodic and opposing pressure fields on the faces of the cantilever causing it to vibrate, thus producing a voltage. Similarly, another design featured a PZT cantilever held parallel to fluid flow with a cylindrical bluff body attached to its tip. Vortex-induced vibrating motion of the cylinder was then transmitted directly to the cantilever [15]. Vortex shedding methods are quite effective in fairly steady free-stream flow; however, they suffer greatly when the upstream flow is spoiled with high levels of turbulence.

\subsection{Proposed approach}

There are two primary differences between existing fluid flow energy harvesting methods and the method presented in this paper. First, neither steady flow conditions nor discrete vortex shedding is assumed to be available. Secondly, rather than having one harvesting device, the proposed design consists of an array of generating elements in the turbulent wake of a bluff body, or in an entirely turbulent fluid flow environment. Robustness and survivability are major concerns when considering the intended environment for the harvester. An attractive feature of this design which directly addresses these concerns is its inherent redundancy. For example: if one element in the array becomes damaged, the device will still produce power. Depending on the size of the array, one damaged element will only contribute to a minor reduction in total output.

Due to the apparently random nature of turbulence, the model presented in this work is separated into two portions. A statistical approach is taken for the TIV portion, while a distributed parameter electromechanical model is developed for the energy harvesting portion. In order to properly measure the turbulence data necessary for the statistical model, hotwire anemometry could not be used. Therefore, it was necessary to use high-sensitivity pressure probes which were designed and built as part of this research. A brief discussion of the pressure probes is included in this paper.

\section{Mathematical modeling}

This section of the paper is intended to introduce and briefly discuss the modeling approach for this work. A more theoretical discussion covering the derivation of this model will be presented by the authors at a later time. Rather than using impractical computationally expensive CFD simulations, statistical methods and random vibration theory are used in this analysis.

\subsection{Spectral statistics}

Modeling turbulent flow is extremely challenging due to its unpredictable nature, and large range of spatial and temporal scales. To help simplify the problem it is sometimes useful to conduct statistical analyses of turbulent flow in the frequency domain. This section discusses the general procedure used for reducing experimentally gathered time-domain data into frequency-domain functions which will be used in a later section to develop the TIV model. For this analysis the turbulent forces acting along the beam are treated as stationary random processes. Note that a random process $p(t)$ is called stationary if its mean, mean square, variance, and standard deviation do not vary with time [16]. A stationary, random pressure in the time domain at a point $z_{a}$ in space $p\left(z_{a}, t\right)$ can be represented in the frequency domain with the well-known Fourier transform.

$$
P\left(z_{a}, \omega\right)=\int_{-\infty}^{\infty} p\left(z_{a}, t\right) \mathrm{e}^{-\mathrm{j} \omega t} .
$$


The imaginary number $\mathrm{j}$ is defined as $\mathrm{j}=\sqrt{-1}$ and $\omega$ is the angular frequency in units of $\mathrm{rad} \mathrm{s}^{-1}$. In order to predict the force exerted by a time-varying pressure on a surface, one must have knowledge of how the pressure at a given point on the surface varies from that of another point at all times. A statistical measure of this is called cross-correlation and the cross-correlation function is defined as

$$
R_{p}\left(z_{a}, z_{b}, \tau\right)=\lim _{T \rightarrow \infty}\left(\frac{1}{2 T}\right) \int_{-T}^{T} p\left(z_{a}, t\right) p\left(z_{b}, t+\tau\right) \mathrm{d} t
$$

where $T$ is the length of the integration interval (or sample time for discrete systems), and $\tau$ is a shift or offset in the time series. Note that if $z_{a}=z_{b}$, then the cross-correlation becomes an autocorrelation. For this analysis we are primarily interested in the pressure power spectral density (PSD) at a given point $z_{a}$ or the cross-power spectral density (CPSD) between two points $z_{a}$ and $z_{b}$. The CPSD function is defined as the Fourier transform of the cross-correlation function and is shown in the following expression,

$$
\begin{aligned}
S_{p}\left(z_{a}, z_{b}, \omega\right)= & \lim _{T \rightarrow \infty}\left(\frac{1}{4 \pi T}\right) \\
& \times\left[\int_{-T}^{T} p\left(z_{a}, t\right) p\left(z_{b}, t+\tau\right) \mathrm{d} t\right] \mathrm{e}^{-\mathrm{j} \omega t} \mathrm{~d} \tau
\end{aligned}
$$

which becomes the PSD if $z_{a}=z_{b}$. Equation (3) is commonly used in spectral analysis; however, care should be taken during implementation. The PSD function is represented in several different forms in the literature, some of which have subtle discrepancies in units that can cause major computational errors. In equation (3) $S_{p}$ is defined as the double-sided PSD function with units $\mathrm{Pa}^{2} \mathrm{~s} \mathrm{rad}^{-1}$. Random vibration theory shows that the mean-squared displacement $\bar{x}^{2}$ of a single degree of freedom oscillator subject to random excitation can be given as [16],

$$
\bar{x}^{2}=\int_{-\infty}^{\infty} S_{x}(\omega) \mathrm{d} \omega=\int_{-\infty}^{\infty}|H(\omega)|^{2} S_{\mathrm{f}}(\omega) \mathrm{d} \omega
$$

where $S_{x}(\omega)$ is the response PSD of the system, $S_{\mathrm{f}}(\omega)$ is the forcing function PSD, and $H(\omega)$ is the complex frequency response function of the oscillator. Applying this approach to a distributed parameter system has many similarities as will be shown in section 2.2.

\subsection{Distributed parameter energy harvesting}

Even though one may desire a large array of piezoelectric grass for practical applications, the model introduced here was developed for a generalized single cantilever case. Beam dynamics were combined with piezoelectric constitutive relationships to provide the electromechanical model. The turbulence-induced forcing function was modeled as a distributed force along the beam length. A statistical method used to estimate the TIV is discussed in section 2.3.

A model for distributed parameter, fully coupled, electromechanical energy harvesting was first introduced by Erturk and Inman for a cantilever beam subject to sinusoidal base excitation [17]. Presented here is a modification of

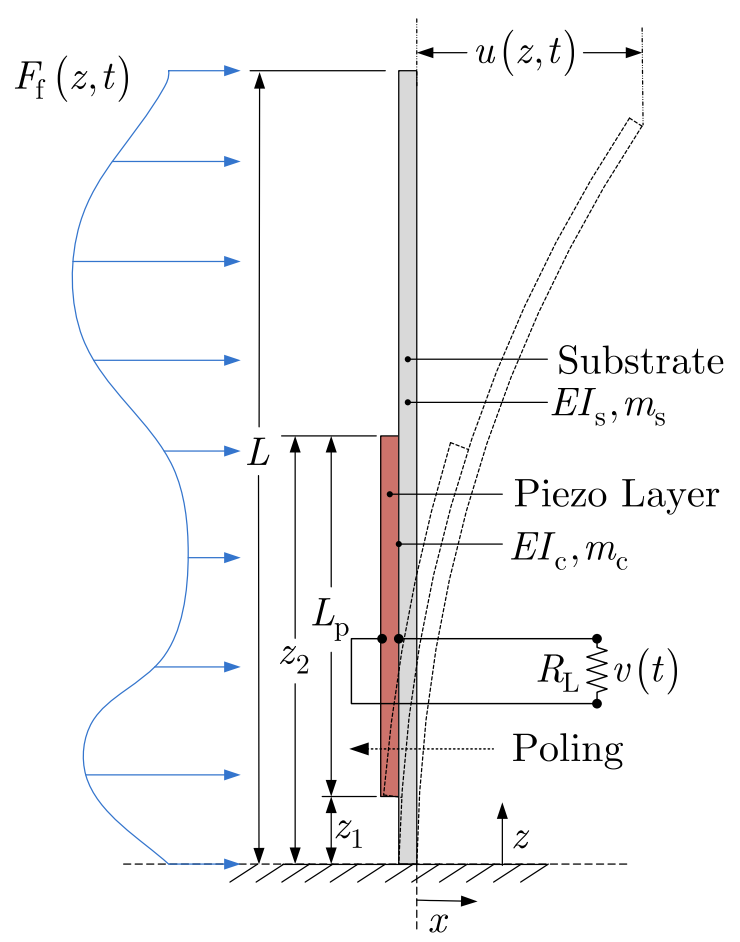

Figure 1. Schematic of a piezoelectric unimorph cantilever exposed to a distributed turbulence-induced force.

their model which includes a distributed turbulence-induced forcing term rather than a base excitation term. The governing differential equation of motion can be given as,

$$
\begin{aligned}
\frac{\partial^{2}}{\partial z^{2}} & {\left[Y I \frac{\partial^{2} u(z, t)}{\partial z^{2}}\right]+\frac{\partial^{2}}{\partial z^{2}}\left[c_{\mathrm{s}} I \frac{\partial^{3} u(z, t)}{\partial z^{2} \partial t}\right]+c_{a} \frac{\partial u(z, t)}{\partial t} } \\
& +m \frac{\partial^{2} u(z, t)}{\partial t^{2}}+\vartheta v(t) \frac{\mathrm{d}}{\mathrm{d} z}\left[\delta\left(z-z_{1}\right)-\delta\left(z-z_{2}\right)\right] \\
= & F_{\mathrm{f}}(z, t)
\end{aligned}
$$

where $Y I$ is the beam bending stiffness, $c_{s}$ and $c_{a}$ are the structural and viscous damping coefficients, respectively, $I$ is the beam area moment of inertia, $m$ is the linear mass density of the beam, $\vartheta$ is the piezoelectric coupling coefficient, $v(t)$ is the induced piezoelectric voltage across a resistive load, and $F_{f}$ is the distributed turbulence-induced forcing function. See [17] for a definition of the piezoelectric coupling term. The Dirac delta function $\delta(z)$ in equation (5) is used to localize the moment induced by the piezoelectric layer along the beam length from $z_{1}$ to $z_{2}$. (See figure 1.) If mass or stiffness over a portion of the cantilever is significantly increased by the piezoelectric material, then one can use the Rayleigh-Ritz method to estimate natural frequencies and mode shapes. Using the method of normal modes, equation (5) is multiplied by the cantilever mode shape $\phi$ and then integrated over the cantilever length to yield the following modal differential equation of motion [16].

$$
\begin{aligned}
& m_{r}\left[\frac{\mathrm{d}^{2} \eta_{r}(t)}{\mathrm{d} t^{2}}+2 \zeta_{r} \omega_{r} \frac{\mathrm{d} \eta_{r}(t)}{\mathrm{d} t}+\omega_{r}^{2} \eta_{r}(t)\right]+\chi_{r} v(t) \\
& =\int_{0}^{L} \phi_{r}(z) F_{f}(z, t) \mathrm{d} z
\end{aligned}
$$


where the total deflection of the cantilever $u(z, t)$ in equation equation (5) is related to the modal displacement $\eta(t)$ and the mode shapes with the following summation series expression.

$$
u(z, t)=\sum_{r=1}^{\infty} \phi_{r}(z) \eta_{r}(t)
$$

In equation (6) $\chi$ is the electromechanical coupling term, $\zeta$ is the modal damping ratio ${ }^{1}$, and $\omega$ is the natural frequency. Derivation of the coupling term $(\chi)$ was shown by Erturk and Inman [17]. Subscripts $r$ and $s$ are integer values that refer to a particular mode of vibration. Note that the mode shapes are not mass normalized; therefore, modal mass for the $r$ th mode of vibration $\left(m_{r}\right)$ must be included in equation (6). If fluid loading effects are significant, a fluid mass term $\mu_{r}$ can simply be added to the modal mass. In order to determine the significance of fluid loading effects, see Blevins [18]. In this work the surrounding fluid is air and the added fluid mass is assumed to be negligible compared to that of the beam ${ }^{2}$. The modal mass is therefore defined as,

$$
m_{r}=\int_{0}^{L} m(z) \phi_{r}^{2}(z) \mathrm{d} z
$$

Assuming a sinusoidal electromechanical response and taking the Fourier transform of equation (6) yields the following expression for modal displacement of the cantilever in the frequency domain.

$$
\eta_{r}(\omega)=\frac{\psi_{r}-\chi_{r} V}{m_{r}\left(\omega_{r}^{2}-\omega^{2}+2 \mathrm{j} \zeta_{r} \omega_{r} \omega\right)} .
$$

The fluid forcing function $\psi$ is also assumed to be sinusoidal; therefore, the Fourier transform of the right-hand side of equation (6) yields,

$$
\psi_{r}(\omega)=\int_{-\infty}^{\infty} \int_{0}^{L} \phi_{r}(z) F_{f}(z, t) \mathrm{e}^{-\mathrm{j} \omega t} \mathrm{~d} z \mathrm{~d} t
$$

which is a frequency-domain representation of the modal fluid forcing term. When the cantilever is exposed to highly turbulent flow, the time-domain forcing function $F_{\mathrm{f}}(z, t)$ required to derive the modal forcing function cannot be defined analytically. Section 2.3 describes a statistical method used to solve for the displacement and power output of a fully elastic cantilever. Fully elastic refers to an uncoupled, simplified model where the backwards coupling piezoelectric effects are neglected. As the focus of this paper is on experimental methods and results, only the uncoupled model will be discussed. It is important to note that the uncoupled model presented in this paper is intended to serve as an estimate for the fully coupled model. Depending on the desired accuracy of the predicted displacement and power output, one may find the uncoupled model sufficient for many applications; however, greatest accuracy will be achieved by considering the fully coupled model. The error attributed to

\footnotetext{
1 Here, the coefficients for structural damping and viscous fluid damping are included as a single estimate for the modal damping ratio.

2 This would not be an acceptable assumption for the same cantilever in water; therefore, a generalized fluid mass loading term $\mu_{r}$ along with an added fluid damping term would be required.
}

neglecting coupling effects for TIV energy harvesting has yet to be investigated.

\subsection{Combined model}

The most challenging aspect of modeling TIV is estimating the forcing function PSD $S_{\mathrm{f}}(\omega)$. Powell was among the first to address these difficulties by developing what is known as the acceptance integral method [19]. This technique is used to provide a measure of how effective a turbulent force is at exciting particular dynamic modes of a structure. One form of the acceptance integral for a 1D case can be given as,

$$
J_{r s}(\omega)=\frac{1}{L S_{p}\left(z_{0}, \omega\right)} \int_{0}^{L} \int_{0}^{L} \phi_{r}(z) S_{p}\left(z, z^{\prime}, \omega\right) \phi_{s}\left(z^{\prime}\right) \mathrm{d} z \mathrm{~d} z^{\prime}
$$

where $S_{p}\left(z_{0}, \omega\right)$ is the pressure PSD at a convenient reference point, $S_{p}\left(z, z^{\prime}, \omega\right)$ is the pressure CPSD along the length of the beam, and $z^{\prime}$ denotes an array of points along the $z$-axis. Au-Yang showed that the total displacement PSD of a structure exposed to turbulent flow can be expressed as [20],

$$
\begin{aligned}
S_{u}(z, \omega)= & \sum_{r=s}^{\infty} \phi_{r}(z)^{2}\left|H_{r}(\omega)\right|^{2} J_{r s}(\omega) \\
& +2 \sum_{r \neq s}^{\infty} \phi_{r}(z) \phi_{s}(z) H_{r}(\omega)^{*} H_{r}(\omega) J_{r s}(\omega)
\end{aligned}
$$

where $H_{r}(\omega)$ is the modal complex frequency response function for the structure ${ }^{3}$. For a cantilever with no piezoelectric coupling, the modal complex frequency response function is,

$$
H_{r}(\omega)=\frac{1}{m_{r}\left(\omega_{r}^{2}-\omega^{2}+2 \mathrm{j} \zeta_{r} \omega_{r} \omega\right)}
$$

The natural frequencies $\omega$, and mode shape functions $\phi$ in equations (6)-(13) were defined one of two ways depending on the cantilever design. For the case of a uniform cantilever, $\omega$ and $\phi$ were defined using classic Euler-Bernoulli beam theory [21]. For the case where mass or stiffness of the cantilever varies along its length, $\omega$ and $\phi$ were defined using a Rayleigh-Ritz approximation.

Analogous to the single degree of freedom random vibration shown in equation (4), the total mean-squared displacement of the distributed parameter model is found by integrating the displacement PSD over the entire frequency range.

$$
\bar{u}^{2}(z)=\int_{-\infty}^{\infty} S_{u}(z, \omega) \mathrm{d} \omega .
$$

In order to estimate the voltage output, it is first assumed that the amplitudes of vibration associated with the cross terms $(r \neq s)$ in equation (12) are significantly less than those for the joint terms $(r=s)$. This is a common assumption used for simplification purposes; however, significant error may result

\footnotetext{
3 The asterisk (*) denotes a complex conjugate of the frequency response function.
} 
if conditions such as those discussed by $\mathrm{Au}$-Yang cannot be met [20]. Preliminary calculations confirmed that cross terms were negligible compared to joint terms for the results presented in this paper.

The general form of the solution for output voltage is assumed to be a convergent series of sinusoidal functions expressed as,

$$
v(t)=\sum_{r=1}^{\infty} V_{r} \mathrm{e}^{\mathrm{j} \omega_{r \mathrm{~d}} t}
$$

where $V_{r}$ is the steady-state voltage amplitude associated with the displacement of the $r$ th mode, and the frequency $\omega_{\mathrm{d}}$ is equal to the damped natural frequency of each mode as shown in equation (17).

$$
\begin{gathered}
\bar{u}_{r}=\sqrt{2} \sqrt{\bar{u}_{r}^{2}}=\sqrt{2 \bar{u}_{r}^{2}} \\
\omega_{r \mathrm{~d}}=\omega_{r} \sqrt{1-\zeta_{r}^{2}} .
\end{gathered}
$$

Note that $\bar{u}^{2}$ is the mean-squared amplitude where $\sqrt{\bar{u}^{2}} \neq$ $\bar{u}$ and the rms amplitude is $\sqrt{\bar{u}^{2}}$; thus, the mean displacement assuming a sinusoidal response is simply $\sqrt{2} u_{\mathrm{rms}}$. Substituting the joint terms from equation (12) into equation (14) and applying the relationship given in equation (16), an expression for total beam displacement can be given as,

$$
\bar{u}(z, t)=\sum_{r}^{\infty}\left[2 \int_{-\infty}^{\infty} \phi_{r}(z)^{2}\left|H_{r}(\omega)\right|^{2} J_{r r}(\omega) \mathrm{d} \omega\right]^{1 / 2} \mathrm{e}^{\mathrm{j} \omega_{r \mathrm{~d}} t}
$$

where the overbar on $u$ denotes a time-averaged function. It is now possible to estimate the voltage output $v(t)$ across the resistive load $R_{\mathrm{L}}$ associated with the total displacement from equation (18) by solving the following differential equation, [17]

$$
v(t)+R_{\mathrm{L}} \frac{\varepsilon_{33}^{\mathrm{S}} b_{\mathrm{p}} L_{\mathrm{p}}}{h_{\mathrm{p}}} \frac{\mathrm{d} v(t)}{\mathrm{d} t}=-R_{\mathrm{L}} \int_{z_{1}}^{z_{2}} d_{31} Y_{\mathrm{p}} h_{\mathrm{pc}} b_{\mathrm{p}} \frac{\partial^{3} \bar{u}(z, t)}{\partial z^{2} \partial t} \mathrm{~d} z
$$

where $d_{31}$ is the piezoelectric constant, $Y_{\mathrm{p}}$ is the Young's modulus of the piezo layer, $h_{\mathrm{pc}}$ is the distance between the central axis of the piezoelectric layer and the neutral axis of the beam, and $\varepsilon_{33}^{\mathrm{S}}$ is the constant strain dielectric permittivity of the piezoelectric layer. From equation (19) it is obvious that the geometry of both the substrate and the piezoelectric layer have a significant effect on voltage output. It is important to note that equation (19) is valid only for piezoelectric unimorph harvesters where it is assumed that the electrodes cover the entire top and bottom surfaces of the piezoelectric layer. The piezoelectric layer edge locations along $z$ are denoted with $z_{1}$ and $z_{2}$ as shown in figure 1 , and $L_{\mathrm{p}}, b_{\mathrm{p}}$ and $h_{\mathrm{p}}$ are the piezoelectric layer length, width and thickness, respectively. If another configuration is desired e.g., a bimorph harvester with constant or segmented electrodes, see Erturk [22].

\section{Experimental methods}

The majority of this work was focused on experimental analysis of several piezoelectric grass harvester prototypes. The most challenging task proved to be attaining accurate turbulence measurements, which required that two custom pressure probes be developed. Details concerning the design and performance of the pressure probes along with measurement techniques, calibration methods, and harvester design will be discussed in this section.

\subsection{Wind tunnel}

A two-stage, open-loop, experimental wind tunnel with continuously variable airspeed control was used to perform all flow experiments. Design and performance details of the wind tunnel are provided by Bilgen (2010) [23]. Existing wind tunnel instrumentation included static pressure ports for free-stream velocity measurements, and an adjustable pitot tube for local steady velocity measurements. A hotwire anemometer, two custom pressure probes, and a Siglab ${ }^{\mathrm{TM}}$ data acquisition system were added to the wind tunnel for these experiments. A virtual control panel implemented with LabVIEW $^{\mathrm{TM}}$ software was used to display, control, and record real-time data.

\subsection{Harvester design}

The type-1 harvester consisted of six generating elements or blades of grass. Each element was a PVDF cantilever from Measurement Specialties, Inc. (model LDT2-028K/L). The type-2 harvester design consisted of four generating elements. Each was constructed by mounting a PZT wafer QuickPack $^{\mathrm{TM}}$ from Mide Technology Corp. (model QP16n) at the root of a spring steel cantilever. A photograph of both harvesters is shown in figure 2 and a summary of the design parameters for each harvester is given in table 1.

Individual load resistors were connected to the electrodes as shown in figure 1 such that each element was an independent circuit. The load resistance $R_{\mathrm{L}}$ used for each type- 1 and type- 2 harvester element was $4.70 \mathrm{M} \Omega$ and $49.2 \mathrm{k} \Omega$, respectively. These resistor values were chosen using the relationship for optimum resistance $R_{\mathrm{L}}=R_{\mathrm{opt}}=$ $1 /\left(\omega_{1} C_{\mathrm{p}}\right)$, where $\omega_{1}$ is the measured, open circuit, first bending mode frequency, and $C_{\mathrm{p}}$ is the measured capacitance of the piezoelectric layer [24]. The measured first bending mode frequency for the type- 1 and type- 2 harvesters was 12.7 Hz and $30 \mathrm{~Hz}$, respectively. Note that this method of determining optimum resistance should only be used when backwards coupling effects are small. See Erturk (2009) for methods of determining the optimum harvester load resistance when piezoelectric coupling effects are considered [22]. The voltage across each resistor was sampled at $2.00 \mathrm{kHz}$ on separate channels with National Instruments data acquisition hardware. In order to allow for convenient rearranging of the individual array elements, magnets were used to secure the cantilevers on a steel grid plate as shown in figure 2 . 


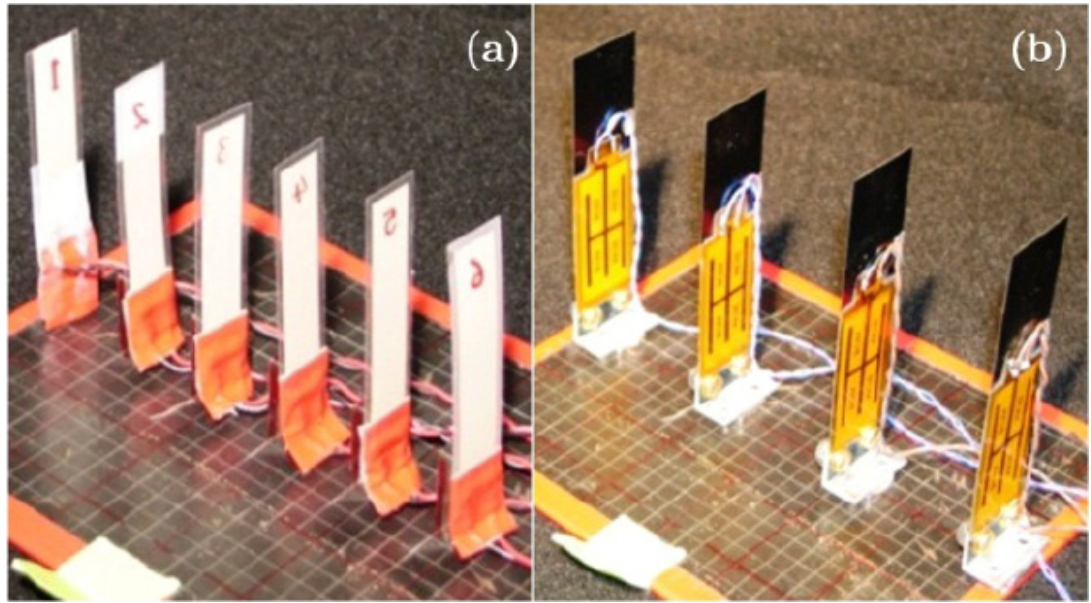

Figure 2. Photograph of inline harvester arrays for both (a) type-1 and (b) type-2 piezoelectric grass harvester prototypes.

Table 1. Summary of design parameters for harvester array elements. (Note: there are several more layers than those listed below for both the PVDF and QuickPack ${ }^{\mathrm{TM}}$ products used in this study. It was found that the added mass and stiffness of the omitted layers had negligible effects on the final results.)

\begin{tabular}{lllccc}
\hline Harvester & Layer & Material & $\begin{array}{l}\text { Length } \\
(\mathrm{mm})\end{array}$ & $\begin{array}{l}\text { Width } \\
(\mathrm{mm})\end{array}$ & $\begin{array}{l}\text { Thickness } \\
(\mu \mathrm{m})\end{array}$ \\
\hline Type-1 & Substrate & Mylar & 72.60 & 16.20 & 178.00 \\
& Piezo & Piezo film & 62.00 & 12.00 & 30.00 \\
Type-2 & Substrate & Steel & 101.60 & 25.40 & 101.60 \\
& Piezo & PZT wafer & 45.97 & 20.57 & 152.40 \\
\hline
\end{tabular}

\subsection{Turbulence measurement}

The existing static and pitot tube pressure sensors installed in the wind tunnel lacked the bandwidth and sensitivity required to make accurate spectral measurements of turbulent flow. Hotwire probes provide excellent bandwidth; however, their accuracy suffers greatly in elevated turbulence intensity levels. For example: a typical single sensor hotwire probe has significant measurement error in turbulence intensities greater than $25 \%$ [25]. It was required that measurements be made in turbulent flow having an intensity well above $25 \%$ where large vortex structures and reversing flows were expected; thus, hotwire anemometry could not be used. According to the literature, the use of pressure probes has proved to be an effective means of performing spectral measurements in high-intensity turbulence; therefore, the authors decided to adopt this approach [26-30].

Two high-sensitivity pressure probes were designed and built to measure low-velocity, high-intensity turbulent flow. Each probe consisted of a differential pressure sensor enclosed such that one port was extended and exposed directly to oncoming flow while the other was isolated within a breathable membrane as illustrated in figure 3. This membrane acted as a filter for the static port to insure that the fluctuating pressure component was measured at the dynamic port only. The pressure sensor in each probe had a differential range of $\pm 249 \mathrm{~Pa}$ with a dynamic response time of $<100 \mu \mathrm{s}$ (All Sensors Corp. model 1-INCH-D-MV). Each probe was designed to have a bandwidth capable of measuring pressure fluctuations associated with the second bending mode of the
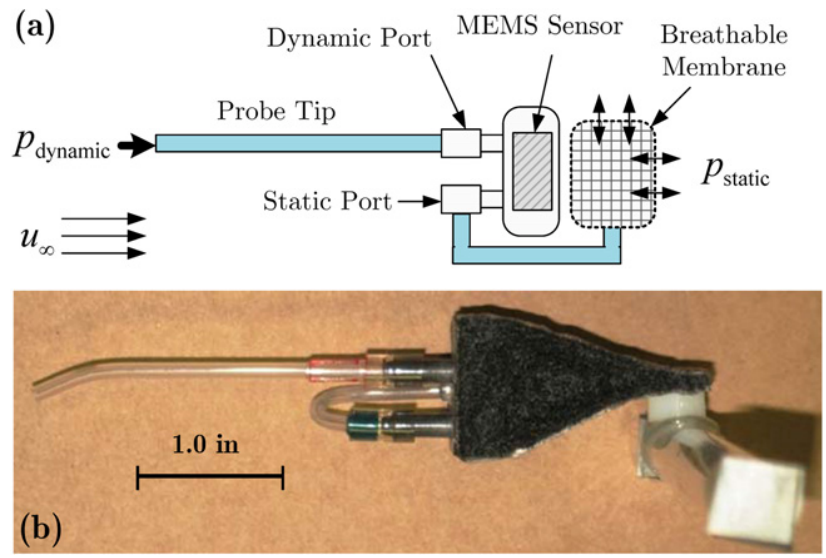

Figure 3. (a) Schematic and (b) photograph of pressure probe used for measuring high-intensity, low-velocity turbulence.

harvesters to be tested. This bandwidth design criteria was set based on observations that showed no significant power contribution from higher modes.

A Dantec 55M01 main unit along with a type 55M10 constant temperature anemometer bridge was used for low-turbulence-intensity spectral measurements. The hotwire probe used was a TSI Model 1201-6 single sensor element. A Siglab ${ }^{\mathrm{TM}}$ data acquisition unit was used to sample and store the time-series sensor voltages at a rate of $12.8 \mathrm{kHz}$. Digital data filtering was performed using a lowpass, fourth-order Butterworth with a cutoff frequency of $2.24 \mathrm{kHz}$. 

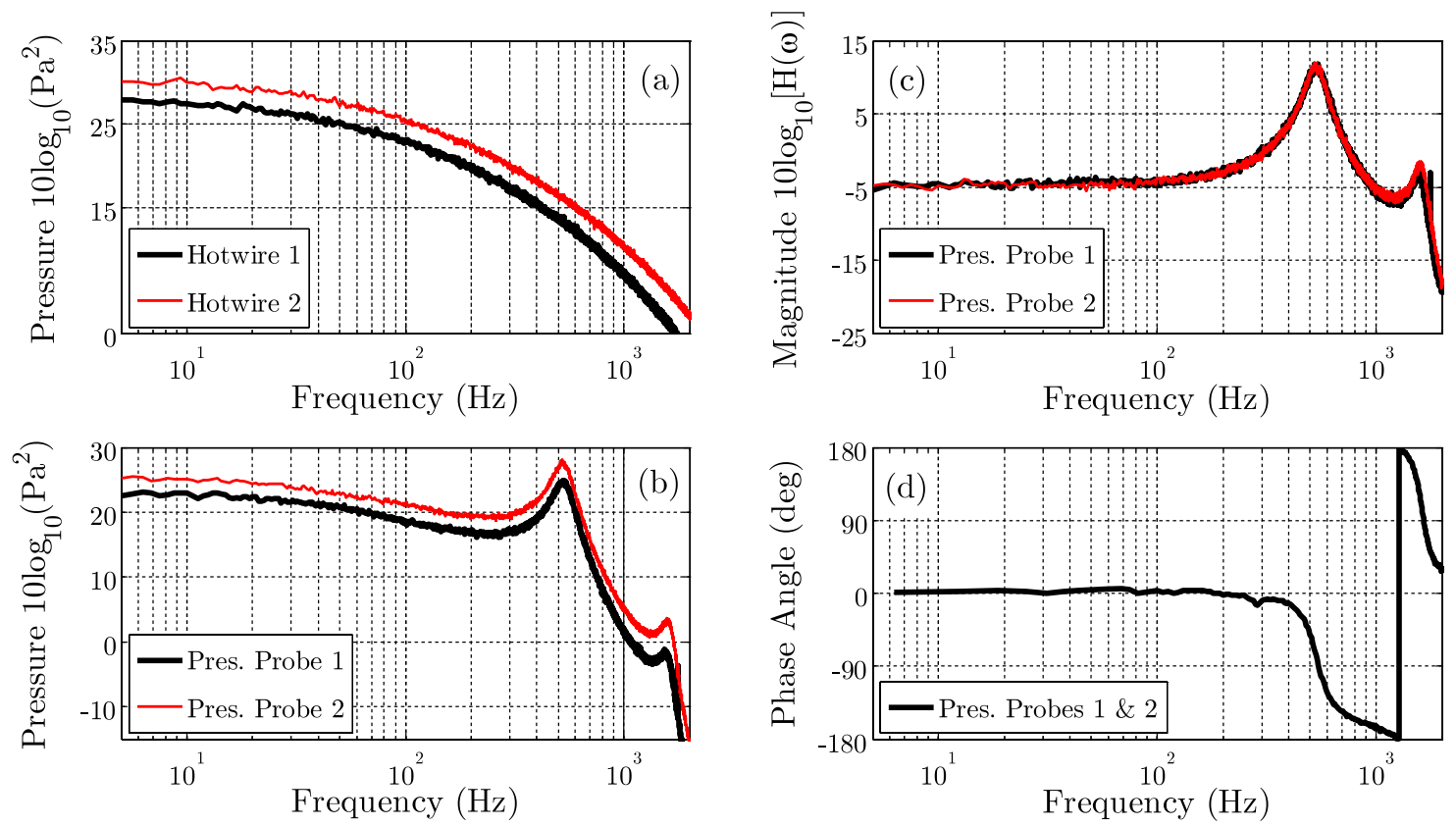

Figure 4. Pressure probe frequency response analysis summary showing power spectral densities measured with (a) hotwire probes and (b) pressure probes. These measurements were used to produce estimates of (c) transfer functions and (d) phase shifts for both pressure probes.

Matlab $^{\mathrm{TM}}$ software was used for all post-processing of the data.

Previously installed wind tunnel instrumentation was used to calibrate the pressure probes in flow having a turbulence intensity less than $1 \%$. The dynamic response characteristics of each probe were found by using a system identification method similar to that used by both Lenherr et al (2011) [30] and Ommen et al (1999) [31]. Grid turbulence having an intensity of approximately $10 \%$ was used as a noise excitation source which was measured with a pressure probe and a hotwire probe simultaneously. The hotwire probe provided what was considered the reference signal, while the pressure probe provided the output signal.

Figures 4(a) and (b) compare the measured PSD functions of the hotwire probe to those of the pressure probes. The higher frequency attenuation in the pressure probe measurements is caused by acoustic resonance within the probe. The measured pressure probe transfer functions and phase angles shown in figures 4(c) and (d) indicate that both pressure probes have a fairly linear response with minimal phase distortion up to approximately $300 \mathrm{~Hz}$. These pressure probe transfer function estimates were confirmed using a model developed by Bergh and Tijdeman [32].

\subsection{Harvester array experiments}

A preliminary experimental study showed that many factors had a significant impact on the harvester array power output. In order to reduce the number of design parameters, it was decided to choose the general design that provided maximum power output as observed in the preliminary study.

Figure 5 shows the general design which was chosen to be an inline array configuration where the array elements are evenly spaced, aligned in the $x$-direction, and offset from the



Air Flow

Figure 5. Inline harvester array configuration.

bluff body in the $y$-direction. Each test consisted of placing a bluff body upstream of the harvester array such that its reference point with respect to the array reference point was known. Free-stream air velocity was then incrementally increased over a range of approximately $1-12 \mathrm{~m} \mathrm{~s}^{-1}$ where $30 \mathrm{~s}$ of data was recorded at a rate of $2.00 \mathrm{kHz}$ at each velocity increment. Both the data acquisition and velocity control were automated with LabVIEW ${ }^{\mathrm{TM}}$. This procedure was repeated while keeping the $y$ offset fixed and varying the $x$ offset.

\subsection{Model validation experiments}

As the model was developed for a single cantilever rather than an array, a model validation study was performed with single cantilevers of various designs. The goal of this study was to measure the turbulent flow without a cantilever present, and then use the model to predict power output from any cantilevered harvester of similar size placed in that flow. 


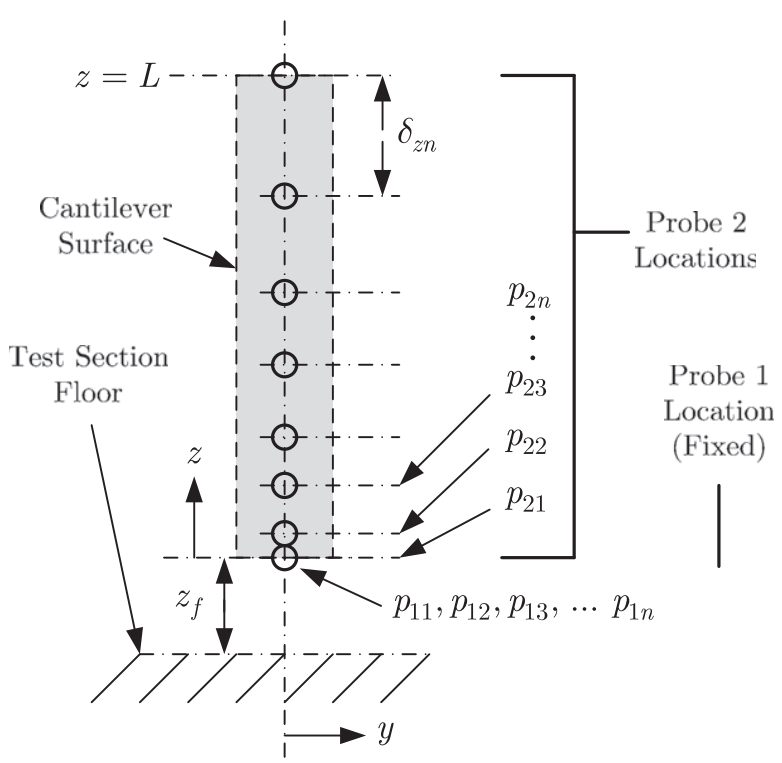

Figure 6. Pressure probe measurement locations relative to the cantilever surface.

For both cases shown, the free-stream velocity, bluff body type and position, and cantilever location were all fixed in order to attain consistent turbulence conditions. The free-stream velocity was set to approximately $11.3 \mathrm{~m} \mathrm{~s}^{-1}$. The bluff body was a rectangular column with dimensions $4.45 \mathrm{~cm} \times 4.45 \mathrm{~cm} \times 10.92 \mathrm{~cm}$ positioned normal to the flow. The pressure probe measurements were made at a location $15.24 \mathrm{~cm}$ downstream and $2.54 \mathrm{~cm}$ offset from the center of the bluff body (i.e. location (15.24, 2.54) $\mathrm{cm}$ using the reference points defined in figure 5). All pressure measurements were made by keeping one probe location fixed $\left(p_{1}\right)$, while the other $\left(p_{2}\right)$ was positioned at points along the length of the beam as shown in figure 6 . Cantilever tip displacement measurements were made with a laser displacement sensor placed inside the wind tunnel downstream of the test section.

\section{Discussion of the results}

\subsection{Harvester array case study}

Figure 7 shows the results of two preliminary case studies that were performed on each of the harvester arrays. Similar procedures as discussed in section 3.4 were followed for each case. The plots in figure 7 illustrate how average power output per harvester array element is related to both flow velocity and harvester position in the near wake of a bluff body. The results show that the type- 2 harvester produced a significantly higher output per cantilever $(1.00 \mathrm{~mW})$ than the type-1 harvester $(1.40 \mu \mathrm{W})$. This drastic difference in output was expected considering the results of an experimental study performed by Shen. Shen showed that the power density of PZT was up to 1000 times greater than PVDF for cantilevered harvesters having a resonant frequency near $100 \mathrm{~Hz}$ [33].

It is clear that there exists an optimum flow velocity and harvester location for both cases. The optimum flow velocity can be predicted with the well-known Strouhal vortex shedding equation, which is given as, [34]

$$
S t=\frac{f_{\mathrm{v}} L_{\mathrm{c}}}{U_{\infty}}
$$

where $f_{\mathrm{v}}$ is the vortex shedding frequency, $U_{\infty}$ is the free-stream velocity, $L_{\mathrm{c}}$ is the characteristic length of the bluff body, and $S t$ is the Strouhal number. It was found that for both cases the absolute maximum power output occurs when the Strouhal shedding frequency matches the natural frequency of the harvester elements $\left(f_{\mathrm{v}}=f_{\mathrm{s}}\right)$. It was also observed that local maxima in power output occurred when the vortex shedding frequency became an integer multiple of the natural frequency of the harvester elements.

\subsection{Single cantilever case study}

The results presented here are from two single cantilever case studies where each case had a different cantilever design. For both cases, the same turbulence conditions were generated in airflow having a mean velocity of $9.8 \mathrm{~m} \mathrm{~s}^{-1}$. The first (case-1) was with a uniform steel cantilever, while the second (case-2) was with a unimorph harvester as shown in figure 1 . Both cantilevers had parameters similar to those given in table 1 for the type- 2 harvester where the active layer was omitted for the case-1 design. Figures 8(a) and (b) shows that the model predictions for tip deflection $\operatorname{PSD} S_{u}(L, \omega)$ agree quite well with measurements for both cases. The results shown in figure 8 (b) are for case- 2 with a load resistance of $30 \mathrm{k} \Omega$. The vortex shedding frequency and structural mode frequencies were intentionally offset to clearly demonstrate the ability of the model to predict both the structural dynamics and the
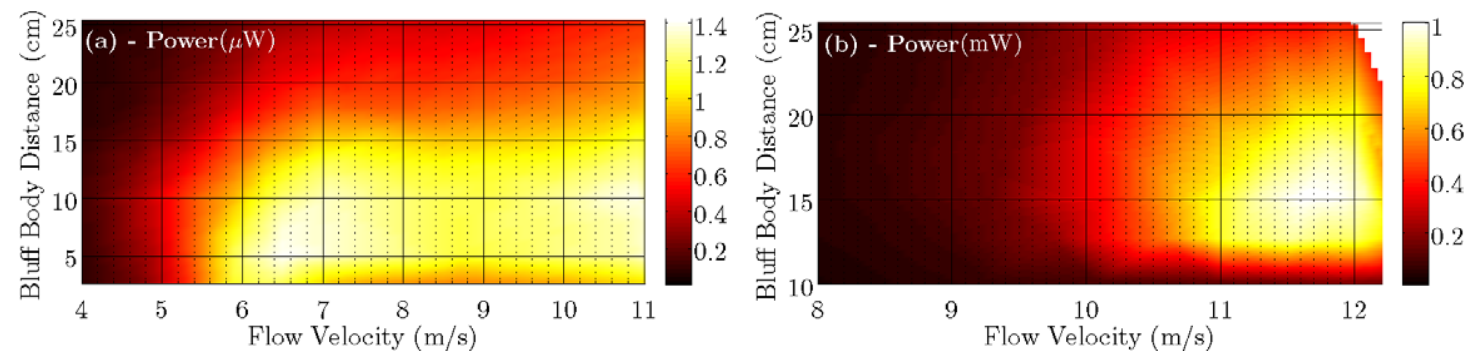

Figure 7. Summary of the results showing power output for two case studies performed with the (a) type-1 and (b) type-2 harvester arrays. 

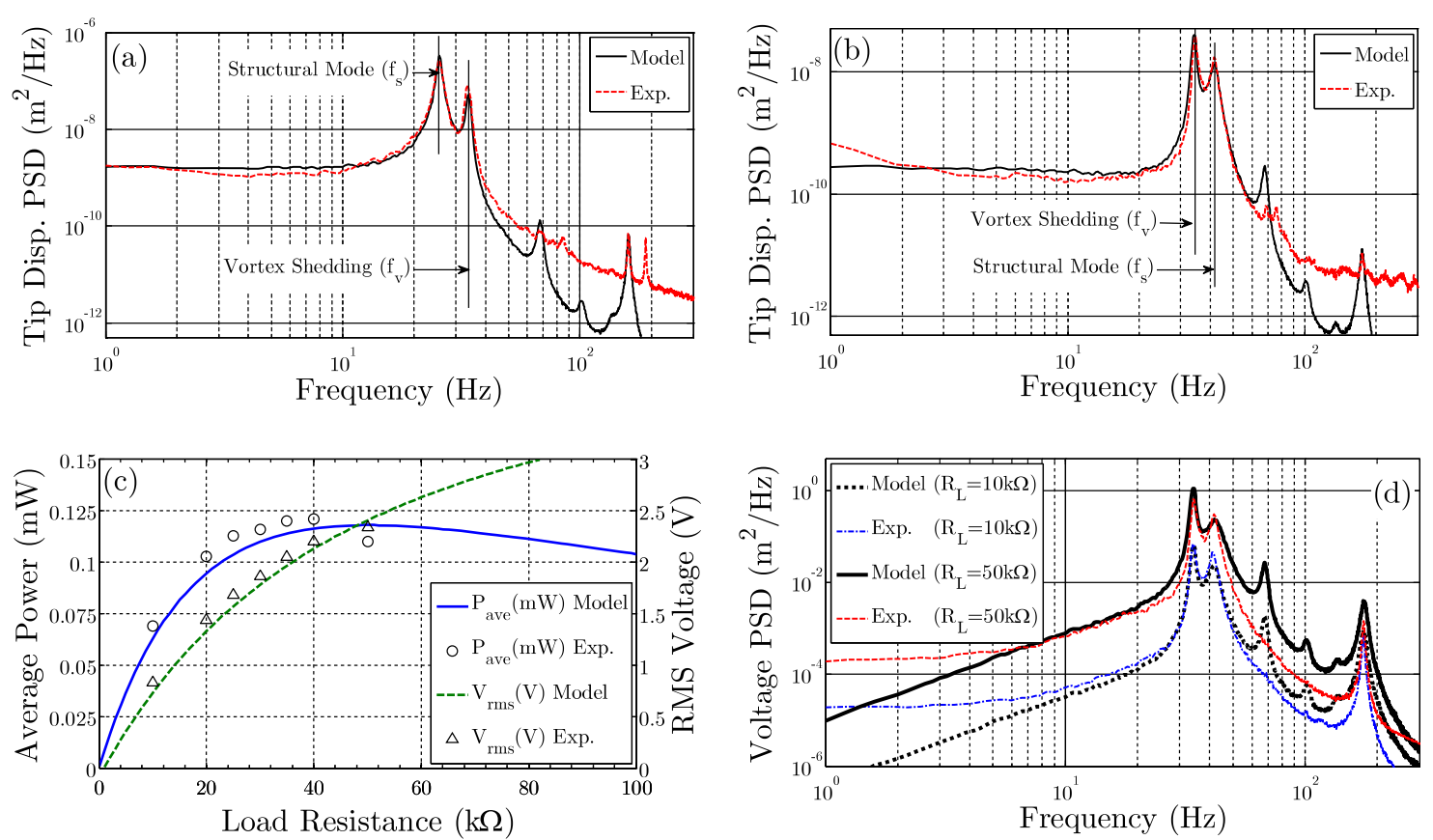

Figure 8. Case study results showing tip displacement PSD for (a) case-1 and (b) case-2, along with case-2 experimental and model results for (c) average power and voltage, and (d) voltage PSD.

turbulent fluid loading. Notice that in figures 8 (a) and (b) the primary vortex shedding frequency $f_{\mathrm{v}}$ was the same for both cases while the structural mode frequency $f_{\mathrm{s}}$ was unique to the cantilever design for each case.

The results in figures $8(\mathrm{c})$ and (d) also demonstrate good agreement between measured and predicted values for voltage and power output. Figure 8(c) shows the power output and rms voltage from the unimorph harvester used in case- 2 for various resistance values ranging from 10 to $50 \mathrm{k} \Omega$. Notice that the estimated optimum resistance of $49.2 \mathrm{k} \Omega$ as discussed in section 3.2 provided a value very near the true optimum resistance in this case study. Figure 8(d) shows the voltage PSD from the case- 2 unimorph for a load resistance of both 10 and $50 \mathrm{k} \Omega$. Again one can see that the model agrees well with measured values at the larger amplitudes. At lower amplitudes however, a majority of the error seen in figures $8(a)$, (b) and (d) can be attributed to sensitivity and bandwidth limitations of the pressure probes. More specifically, the pressure probe output sensitivity to pressure fluctuation is overcome by the electrical noise floor, and at higher frequencies the probes are nearing their bandwidth limit of $300 \mathrm{~Hz}$ as discussed in section 3.3 .

\section{Conclusions}

Extensive experimental work has been done on several piezoelectric grass prototypes. It was shown that the PZT harvester array (type-2) was able to achieve a power output of $1.0 \mathrm{~mW}$ per cantilever with a mean airspeed of $11.5 \mathrm{~m} \mathrm{~s}^{-1}$. The similarly sized PVDF harvester array (type-1) was expected to produce significantly less power, but was still able to achieve an output of $1.2 \mu \mathrm{W}$ per cantilever at $7 \mathrm{~m} \mathrm{~s}^{-1}$. From an application standpoint, note that the PZT harvester produced nearly 1000 times the output for approximately ten times the cost compared to the PVDF harvester. However, when considering long-term deployment in an uncontrolled environment, the soft, flexible PVDF design is much less susceptible to damage than the brittle PZT design.

Harvester array results show that an optimum turbulence condition for maximum power output exists. It was observed that these ideal harvesting conditions are functions of both flow velocity and harvester location downstream of a bluff body. An estimate of the optimum harvester design can be attained by matching the natural frequency of the harvester to the primary vortex shedding frequency of the bluff body. While the array studies presented here were strictly experimental, these results provide valuable insight for the future development of mathematical models for large harvester arrays.

Two high-sensitivity pressure probes were successfully designed and implemented. These probes provided accurate measurements of high-intensity ( $>80 \%)$, low-velocity (1-20 $\left.\mathrm{m} \mathrm{s}^{-1}\right)$ turbulent air flow. These measurements were used in the statistical portion of the model to predict the TIV of a cantilever beam. A noise excitation, system identification method was used to achieve estimates of transfer functions and phase distortion caused by acoustic resonance within the probes. The linear response bandwidth of both probes was designed to be $300 \mathrm{~Hz}$.

A combination of analytical and statistical modeling techniques for predicting single cantilever TIV energy harvesting was presented. The results of two case studies were discussed, and demonstrated excellent agreement between the model predictions and experimental data. According to the literature, this paper introduces the first experimentally validated TIV energy harvesting model. The harvester designs in this paper were not optimized, yet were still able to achieve among the highest power output compared to 
related harvesting methods. The authors hope this work will encourage and inspire other researchers to further explore the possibilities of harvesting energy from highly turbulent fluid flow.

\section{Acknowledgments}

The authors gratefully acknowledge the support of Science Applications International Corporation (SAIC) for funding this research through the NSF I/UCRC Center for Energy Harvesting Materials and Systems at Virginia Tech.

\section{References}

[1] Hobeck J D and Inman D J 2011 Energy harvesting from turbulence-induced vibration in air flow: artificial piezoelectric grass concept ASME Conf. Proc. 2011 637-46

[2] Au-Yang M K 2001 Flow-Induced Vibration of Power and Process Plant Components: A Practical Workbook (New York: ASME Press)

[3] Akaydin H D, Elvin N and Andreopoulos Y 2010 Energy harvesting from highly unsteady fluid flows using piezoelectric materials J. Intell. Mater. Syst. Struct. 211263

[4] Myers R, Vickers M, Kim H and Priya S 2007 Small scale windmill Appl. Phys. Lett. 90054106

[5] Priya S 2005 Modeling of electric energy harvesting using piezoelectric windmill Appl. Phys. Lett. 87184101

[6] Pankonien A M and Ounaies Z 2010 Piezoelectric artificial kelp for energy harvesting ASME Conf. Proc. 2010 223-32

[7] Hobbs W B and Hu D L 2012 Tree-inspired piezoelectric energy harvesting J. Fluid Struct. 28 103-14

[8] Bryant M and Garcia E 2009 Development of an aeroelastic vibration power harvester Proc. SPIE 7288728812

[9] Bryant M and Garcia E 2009 Energy harvesting: a key to wireless sensor nodes Proc. SPIE 7493 74931W

[10] Jung H J, Lee S W and Jang D D 2009 Feasibility study on a new energy harvesting electromagnetic device using aerodynamic instability Magnetics, IEEE Trans. 45 4376-9

[11] Shimizu E, Isogai K and Obayashi S 2008 Multiobjective design study of a flapping wing power generator J. Fluid Eng. 130021104

[12] De Marqui C et al 2010 Piezoaeroelastic modeling and analysis of a generator wing with continuous and segmented electrodes J. Intell. Mater. Syst. Struct. 21 983-93

[13] Bernitsas M M et al 2008 Vivace (vortex induced vibration aquatic clean energy): a new concept in generation of clean and renewable energy from fluid flow $J$. Offshore Mech. Arctic Eng. 130041101

[14] Pobering S 2011 Energy harvesting under induced best conditions Structural Dynamics and Renewable Energy vol 10, ed T Proulx (New York: Springer) pp 239-45

[15] Akaydin H D, Elvin N and Andreopoulos Y 2010 Experimental study of a self-excited piezoelectric energy harvester ASME Conf. Proc. 2010 179-85
[16] Newland D E 1975 An Introduction to Random Vibrations and Spectral Analysis (London: Longman)

[17] Erturk A and Inman D J 2008 A distributed parameter electromechanical model for cantilevered piezoelectric energy harvesters J. Vib. Acoust. 130041002

[18] Blevins R D 1977 Flow-Induced Vibration vol 1 (NewYork: Van Nostrand-Reinhold) p 377

[19] Powell A 1958 On the fatigue failure of structures due to vibrations excited by random pressure fields J. Acoust. Soc. Am. 30 1130-5

[20] Au-Yang M 2000 Joint and cross acceptances for cross-flow-induced vibration-part II: charts and applications J. Press. Vessel Technol. 122 355-61

[21] Blevins R D 1979 Formulas for Natural Frequency and Mode Shape (NewYork: Van Nostrand-Reinhold)

[22] Erturk A 2009 Electromechanical modeling of piezoelectric energy harvesters PhD Dissertation Virginia Polytechnic Institute \& State University

[23] Bilgen O 2010 Aerodynamic and electromechanical design, modeling and implementation of piezocomposite airfoils PhD Dissertation Virginia Polytechnic Institute \& State University

[24] Lu F, Lee H and Lim S 2004 Modeling and analysis of micro piezoelectric power generators for micro-electromechanical-systems applications Smart Mater. Struct. 1357

[25] Bruun H H 1995 Hot-Wire Anemometry: Principles and Signal Analysis (Oxford: Oxford University Press)

[26] Ainsworth R, Miller R, Moss R and Thorpe S 2000 Unsteady pressure measurement Meas. Sci. Technol. 111055

[27] Chue S 1975 Pressure probes for fluid measurement Prog. Aerosp. Sci. 16 147-223

[28] Kupferschmied P, Gossweiler C and Gyarmathy G 1994 Aerodynamic fast-response probe measurement systems: state of development, limitations and future trends J. Turbomach. 122 505-16

[29] Jezdinsky V 1966 Measurement of turbulence by pressure probes AIAA J. 42072

[30] Lenherr C, Kalfas A I and Abhari R S 2011 High temperature fast response aerodynamic probe J. Eng. Gas Turbines Power 133011603

[31] van Ommen J R, Schouten J C, vander Stappen M L M and van den Bleek C M 1999 Response characteristics of probe-transducer systems for pressure measurements in gas-solid fluidized beds: how to prevent pitfalls in dynamic pressure measurements Powder Technol. 106 199-218

[32] Bergh H and Tijdeman H 1965 Theoretical and Experimental Results for the Dynamic Response of Pressure Measuring Systems (Amsterdam: National Aero- and Astronautical Research Institute)

[33] Shen D, Choe S Y and Kim D J 2007 Analysis of piezoelectric materials for energy harvesting devices under high-g vibrations Japan. J. Appl. Phys. 466755

[34] Crowe C T, Elger D F, Roberson J A and Williams B C 2008 Engineering Fluid Mechanics (New York: Wiley) 